\title{
Cardiac amyloidosis the contribution of images for diagnosis
}

\begin{abstract}
The restrictive pathologies are a group of diseases of low prevalence. Cardiac amyloidosis is considered to be the prototype of the infiltrative form of restrictive cardiomyopathies. The myocardial involvement is frequent, even at the first appearance of the disease and it has a poor prognosis; and although pleural involvement is less frequent, its presence determines a much more torpid evolution than isolated cardiac presentations. The main problem that these pathologies concern is their late recognition and the consequences this implies in the patient evolution. Nowadays, cardiac images tools may facilitate the diagnosis, allowing an early detection. We share an unusual case of amyloidosis with pleural involvement, where the results of the echocardiogram and the cardiac magnetic resonance allowed an approach to an accurate diagnostic.
\end{abstract}

Volume I 3 Issue I - 2020

\author{
Juan I Cotella, Dr Aldo Prado \\ Department of Echocardiography, CPC - Private De Cardiology \\ Center, Argentina
}

\begin{abstract}
Correspondence: Juan I Cotella, Department of Echocardiography, CPC - Private De Cardiology Center, Argentina, Fax 5438|431।009, Tel 5493874869170, Email juanicotella@gmail.com
\end{abstract}

Received: January 24, 2020 | Published: February 18, 2020

Keywords: amyloidosis, restrictive, infiltrative, magnetic resonance, echocardiogram

Abbreviations: IR, inversion recovery; LV, left ventricle; CPR, cardiopulmonary resuscitation; MRI, magnetic resonance imaging

\section{Introduction}

The group of diseases associated to abnormal diastolic function with restrictive pattern is very rare in clinical practice. ${ }^{1}$ Amyloidosis represents one of the most characteristic prototypes of these pathologies. Ambiguity in clinical practice leads to a late and inaccurate diagnosis, performed correctly in less than $20 \%$ of patients. ${ }^{2}$ Cardiac image studies are important for the early diagnosis; the echocardiogram is one of the main tools in the diagnostic algorithm. The case we developed hereafter is an example of a common setting of the appearance of this pathology, revealing that if there is no clinical suspicion, the probabilities to achieve an accurate diagnosis are low.

\section{Case presentation}

The case is about a female patient of 65 years old, obese, with dyslipidemia, hypertension, asthma, with a background of dyspnea NYHA class III of 3 months of evolution and a weight loss of $10 \mathrm{~kg}$. During this period, she referred many medical visits due to these symptoms. In one of them, an electrocardiogram was performed, with a pattern of QS from V1 to V6 observed on it. This motivated to perform an echocardiogram, reporting concentric hypertrophy and mild pericardial effusion. Then, a myocardial perfusion imaging was performed, showing a severe anterior and lateral ischemia. Finally, a coronary angiography was performed, exhibiting absence of coronary stenosis.

Subsequently, she was admitted to our institution with a clinic diagnosis of acute heart failure, Stevenson B. A chest X-ray showed bilateral pleural effusion, grade II. During the admission, the only significant finding was mild anemia $(\mathrm{Hb} 11,1 \mathrm{~g} / \mathrm{dl})$. A transthoracic echocardiogram (Figure 1 \& Video 1) was done showing normal left ventricle diameters, LV ejection fraction $47 \%$, mild concentric hypertrophy, biatrial enlargement, mild to moderate mitral regurgitation, pseudo normal ventricular filling pattern, e $4 \mathrm{~cm} /$ sec, E/e' ratio greater than 18 , moderate tricuspid insufficiency, an estimate pulmonary artery systolic pressure of $55 \mathrm{mmHg}$ and mild pericardial effusion. The presumptive diagnosis of infiltrative restrictive cardiomyopathy was assumed. A myocardial 3D strain imaging with speckle tracking was done, showing normal longitudinal strain of apical segments and a strong impaired values of basal and mid segments (-16 to -28 at apical segments vs 4 to -4 at basal level) (Figure 2C). The patient improved clinically with diuretics and vasodilators according to local guidelines. Nevertheless, during the third day of hospitalization she experiences a sudden death episode, with ventricular tachycardia documented in the telemetric system, with an adequate response to cardiopulmonary resuscitation (CPR).

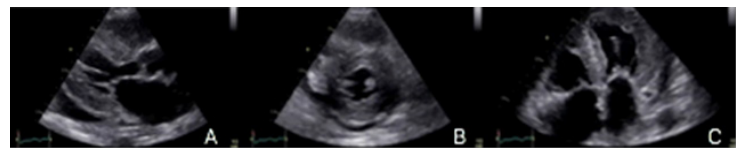

Figure I We can see a parasternal long axis view (A), short axis at mid ventricular level (B) and 4 chambers (C), showing mild hypertrophy $(12 \mathrm{~mm})$ with biatrial enlargement and mild pericardial effusion (White arrow).
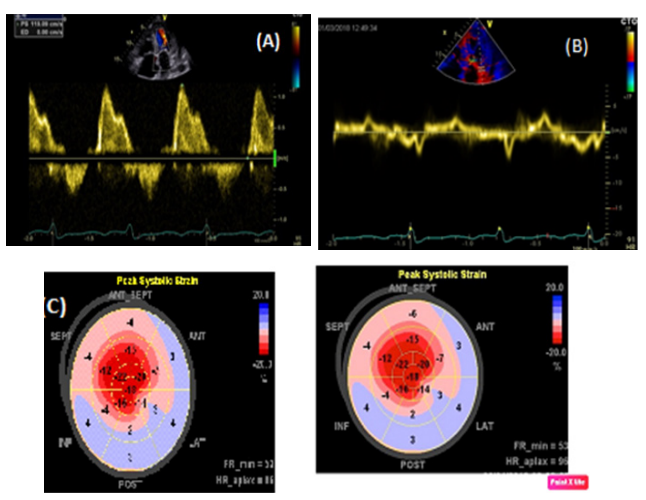

Figure 2 diastolic mitral flows with pseudo normal pattern $(A)$. E' wave plainly decreased to the side mitral ring level (B). Global longitudinal strain with normal values at apical level and diminished in mid and basal segments (C). 


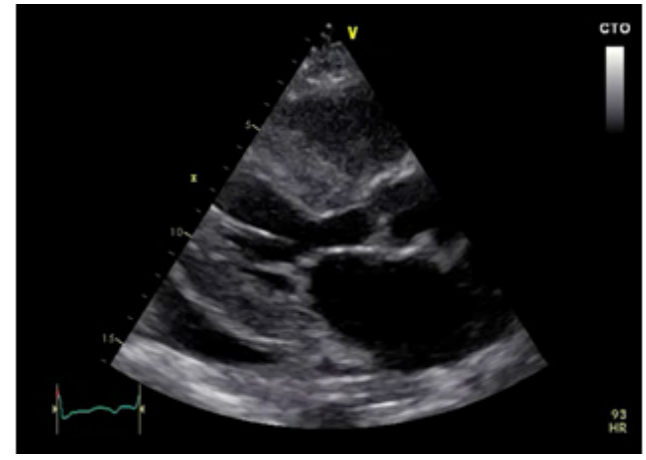

Video I parasternal long axis, shot and apical axis view. 2D, color doppler, showing mild hypertrophy with biatrial enlargement, mild pericardial effusion, mild to moderate mitral regurgitation, moderate tricuspid regurgitation, inferior cava vein dilated.

An MRI (Figure 3) was performed, reporting during inversion recovery sequences (IR) of late gadolinium enhancement the impossibility to diminish properly the left ventricle due to its global involvement and the hypo-intensity signal at intra-cavitary level, despite performing IR sequence 5 (five) minutes after contrast injections. During look Locker sequence, an impairment of more than $50 \%$ of the left ventricle myocardium was found. Leading the diagnostic suspicion of amyloidosis, a pleural puncture was performed revealing $6 \mathrm{~g} / \mathrm{dl}$ of protein in the exudate with a high number of lymphocytes, polymorph nuclear leucocytes, plenty of atypical mesothelial cells and a low number of atypical plasmatic cells. Bacterial and mycobacterial culture were negative. Then, a fine needle aspiration biopsy of abdominal fat was made, giving a positive result for amyloid. The patient was moved to the haemato-oncology unit in order to evaluate an alternative treatment and she died 22days later.

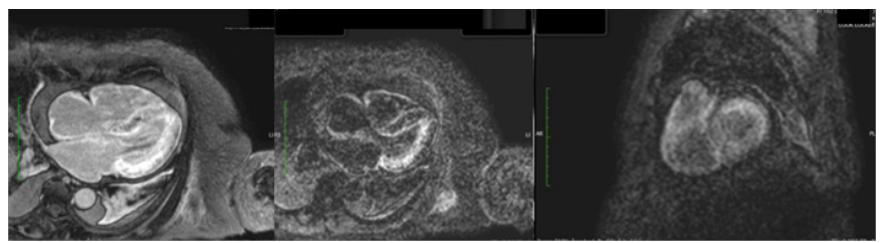

Figure 3 Sequence of late enhancement in short axis and in the four chambers. Impossibility to dim the myocardium properly due to global involvement, together with intracavitary hypo-intensity. Sub-endocardial and sub-epicardial diffuse involvement in left ventricle, as well as in atrial myocardium and intraatrial septum.

\section{Discussion}

Cardiac amyloidosis was considered an uncommon disease, however, nowadays; there is a suspicion that the low prevalence is associated to the deficiency or delay in the diagnosis. ${ }^{6}$ These mistakes are multifactorial, especially due to the appearance diversity, the early low suspicion and the lack of proper diagnostic strategies.

Amyloidosis means a group of diseases of different etiologies with a common characteristic: the amorphous material deposit at extracellular level, affecting the heart, liver and other tissues. Even though clinical and image characteristics of different types of amyloidosis have a lot in common, therapeutic strategies and prognosis are usually different in each of them.
Among the ones affecting the myocardial tissue more frequently, there are two types: those associated to light chains (AL) production, and those associated to transthyretin (TTR). Knowing the difference between them helps to an early diagnosis and, of course, an individual therapeutic approach. In the US, there is around 3,000 new cases yearly. ${ }^{4}$ The clinical appearance usually varies, being heart condition the main defining prognosis. Myocardial involvement is usually shown by symptoms as heart failure and atrial and ventricular arrhythmias.

The physio-pathological mechanism, by which the systolic and diastolic dysfunction is built, is determined by both the direct amyloid deposit and the "toxic" effect of the deposited and circulating protein parent. ${ }^{5}$ The presence of heart failure of underlying cause, associated to hepatomegaly and proteinuria should be the first diagnostic suspicious. Considering this situation, an electrophoretic study must be performed to quantify the presence of protein chains (kappa y lambda). The tissue biopsy, to confirm the amyloid deposit, is an essential element for diagnosis able to be obtained by aspiration of abdominal fatty tissue. The bone marrow puncture allows establishing the specific clonal diagnosis. The endomyocardial biopsy is only necessary in less than $15 \%$ of patients; however, the specificity is $100 \%{ }^{6,7}$

Imaging studies are clearly useful; nevertheless, they do not show specific data to differentiate the diverse types of diseases. Classic data include normal size ventricles with mild hypertrophy, biatrial enlargement, changes in the ventricular filling pattern (pseudo normal or restrictive pattern) and pericardial effusion. The myocardial assessment with longitudinal strain shows a very important characteristic pattern for the diagnosis, characterized by strain values diminished to basal level and mid segments, with normal values of apical segments. These findings may be present in absence of ventricular motility disorders, so the benefit is understandable ${ }^{8}$ Our patient presented all these features.

With regards to electrocardiographic evidences, we recalled that in our case a pseudo-infarction image in the front-side face was found. These manifestations are usually present in less than the half of patients. The presence of pleural effusion described in our patient is usually present in less than $6 \%$ of patient with myeloma, nevertheless, incidence of pleural infiltration is of less than $0,8 \%,{ }^{10,11}$ being associated to a mean of livelihood of 1,5-3months. ${ }^{12}$

AL type amyloidosis diagnosis may be performed in $95 \%$ of cases where the combination of light chain findings in plasma and urine are present, through electrophoretic studies of immunofixation and fatty tissue aspiration. Even then, the previous diagnosis is important and, at this point, image methods are defining. Whereby, in light of the distinctive echocardiographic findings, including the longitudinal strain, or the changes observed in MRI studies, added to the detection of monoclonal protein expression, there is an approach to the diagnosis to a large extent, without being necessary the bone marrow biopsy puncture nor the endomyocardial biopsy.

Annual mortality of patients with amyloidosis and heart involvement reach yearly numbers of $24 \%$, and in those cases where death is present in the first six months, half of them are sudden death. ${ }^{13,14}$ The grade of heart condition is a determining prognosis, and in this sense, MRI is a heavy weight prognosis attribute through determination of extracellular involvement grade. ${ }^{15}$ 


\section{Conclusion}

Cardiac amyloidosis is considered an uncommon disease but should be consider as a possibility in some patients admitted due to cardiac heart failure without a clear etiology. Most typical echocardiography findings include normal size ventricles with mild hypertrophy, biatrial enlargement and changes in the ventricular filling pattern. Global longitudinal strain has been shown as a very useful echo tool providing a very important characteristic pattern for the diagnosis.

\section{Acknowledgments}

None.

\section{Conflicts of interest}

Author declares that there are no conflicts of interest towards publication.

\section{Funding}

None.

\section{References}

1. Elliott P, Andersson B, Arbustini E, et al. Classification of the cardiomyopathies: a position statement from the European Society Of Cardiology Working Group on Myocardial and Pericardial Diseases. Eur Heart J. 2008;29(2):270-276.

2. Maurer MS, Elliott P, Comenzo R, et al. Addressing common questions encountered in the diagnosis and management of cardiac amyloidosis. Circulation. 2017;135(14):1357-1377.

3. Lousada I, Comenzo RL, Landau H, et al. Light chain amyloidosis: patient experience survey from the Amyloidosis Research Consortium. Adv Ther. 2015;32(10):920-928.

4. Kyle RA, Linos A, Beard CM, et al. Incidence and natural history of primary systemic amyloidosis in Olmsted County, Minnesota, 1950 through 1989. Blood. 1992;79(7):1817-1822.
5. Brenner DA, Jain M, Pimentel DR, et al. Human amyloid genic light chains directly impair cardiomyocyte function through an increase in cellular oxidant stress. Circ Res. 2004;94(8):1008-1010.

6. Gertz MA. Immunoglobulin light chain amyloidosis: 2011 update on diagnosis, risk- stratification, and management. Am J Hematol. 2011;86(2):180-186.

7. Maleszewski JJ. Cardiac amyloidosis: pathology, nomenclature, and typing. Cardiovasc Pathol. 2015;24(6):343-350.

8. Bellavia D, Abraham TP, Pellikka PA, et al. Detection of left systolic dysfunction in cardiac amyloidosis with strain rate echocardiography. $J$ Am SocEchocardiogr. 2007;20(10):1194-1202.

9. Murtagh B, Hammill SC, Gertz MA, et al. Electrocardiographic findings in primary systemic amyloidosis and biopsy-proven cardiac involvement. Am J Cardiol. 2005;95(4):535-537.

10. Oudart JB, Maquart FX, Semouma O, et al. Pleural effusion in a patient with multiple myeloma. Clin Chem. 2012;58(4):672-674.

11. Rodríguez JN, Pereira A, Martínez JC, et al. Pleural effusion in multiple myeloma. Chest. 1994;105(2):622-624.

12. Riveiro V, Toubes ME, Lama A, et al. Characteristics of patients with myelomatous pleural effusion. A systematic review. Rev Clin Esp. 2018;218(2):89-97.

13. Falk RH, Alexander KM, Liao R, et al. AL (light-chain) cardiac amyloidosis: a review of diagnosis and therapy. $\mathrm{J} \mathrm{Am}$ Coll Cardiol. 2016;68(12):1323-1341.

14. Muchtar E, Gertz MA, Kumar SK, et al. Improved outcomes for newly diagnosed AL amyloidosis between 2000 and 2014: cracking the glass ceiling of early death. Blood. 2017;129(15):2111-2119.

15. Banypersad SM, Fontana M, Maestrini V, et al. T1 mapping and survival in systemic light-chain amyloidosis. Eur Heart J. 2015;36(4):244-251. 